\title{
SOME ASYMPTOTIC FORMULAE FOR THE MOCK THETA SERIES OF RAMANUJAN $\left({ }^{1}\right)$
}

\author{
BY

\section{LEILA A. DRAGONETTE}

1. Introduction. In his last letter to Hardy $[1 ; 3]\left({ }^{2}\right)$, Ramanujan described a set of functions defined by Eulerian series inside the unit circle, possessing asymptotic representations at their essential singularities of the same order of precision as those known for the theta functions. As an example of the latter, consider the generating function of the partition numbers $p(n)$,

$$
P(q)=\sum_{n=0}^{\infty} p(n) q^{n}=\prod_{m=1}^{\infty}\left(1-q^{m}\right)^{-1}=\left[-i q^{1 / 8} \vartheta_{1}\left(\frac{\tau}{2} / \frac{3 \tau}{2}\right)\right]^{-1}
$$

where $q=e^{\pi i \tau}$ and $\vartheta_{1}(v / \tau)=\vartheta_{1}(v, q)$. This function, which may also be written in Eulerian form,

$$
\begin{aligned}
P(q)=1 & +\frac{q}{(1-q)^{2}}+\frac{q^{4}}{(1-q)^{2}\left(1-q^{2}\right)^{2}} \\
& +\frac{q^{9}}{(1-q)^{2}\left(1-q^{2}\right)^{2}\left(1-q^{3}\right)^{2}}+\cdots,
\end{aligned}
$$

possesses an asymptotic representation in closed exponential form in the neighborhood of rational points of the unit circle, derived from the theory of linear transformations: if $q=e^{2 \pi i h / k-2 \pi t / k}$ and real $t \rightarrow 0$,

$$
P\left(e^{2 \pi i h / k-2 \pi t / k}\right)=\omega_{h, k} t^{1 / 2} e^{(\pi / 12 k)(1 / t-t)}+o(1)
$$

where $\omega_{h, k}$ is a certain root of unity.

On the other hand, not every function in Eulerian form behaves in a closed form at the essential singularities on its natural boundary. For example, if $q=e^{-\pi t}$ and $t \rightarrow 0+$, it can be shown that

$$
\begin{aligned}
1+\frac{q}{(1-q)^{2}}+\frac{q^{3}}{(1-q)^{2}\left(1-q^{2}\right)^{2}}+\frac{q^{6}}{(1-q)^{2}\left(1-q^{2}\right)^{2}\left(1-q^{3}\right)^{2}}+\cdots \\
=\left(\frac{t}{2\left(5^{1 / 2}\right)}\right)^{1 / 2} \exp \pi\left(\frac{1}{5 t}+a_{1} t+a_{2} t^{2}+\cdots+O\left(a_{j} t^{3}\right)\right) .
\end{aligned}
$$

This counter-example has been discussed by Watson [3].

Received by the editors November 9, 1951.

(1) Presented to the Faculty of the Graduate School of the University of Pennsylvania in partial fulfillment of the requirements for the degree of Doctor of Philosophy, 1951.

(2) Numbers in brackets refer to the bibliography at the end of the paper. 
If a function $f(q)$ in Eulerian form has an infinity of essential singularities, at which the asymptotic representation closes as neatly as for $P(q)$, and if $f(q)$ is not the sum of an ordinary theta function and a (trivial) function which is $O(1)$ at all the singular points, then $f(q)$ is called a mock theta function.

As examples Ramanujan presents three sets of mock theta functions (of third, fifth, and seventh order) with specific identities relating the functions of each set (except the seventh order) to each other and to ordinary theta series. The complete list of functions of third order follows:

$$
\begin{aligned}
f(q) & =\sum_{n=0}^{\infty} \frac{q^{n^{2}}}{(1+q)^{2}\left(1+q^{2}\right)^{2} \cdots\left(1+q^{n}\right)^{2}}, \\
\phi(q) & =\sum_{n=0}^{\infty} \frac{q^{n^{2}}}{\left(1+q^{2}\right)\left(1+q^{4}\right) \cdots\left(1+q^{2 n}\right)}, \\
\psi(q) & =\sum_{n=1}^{\infty} \frac{q^{n^{2}}}{(1-q)\left(1-q^{3}\right) \cdots\left(1-q^{2 n-1}\right)}, \\
\chi(q) & =\sum_{n=0}^{\infty} \frac{q^{n^{2}}}{\left(1-q+q^{2}\right)\left(1-q^{2}+q^{4}\right) \cdots\left(1-q^{n}+q^{2 n}\right)}, \\
\omega(q) & =\sum_{n=0}^{\infty} \frac{q^{2 n(n+1)}}{(1-q)^{2}\left(1-q^{3}\right)^{2} \cdots\left(1-q^{2 n+1}\right)^{2}}, \\
\nu(q) & =\sum_{n=0}^{\infty} \frac{q^{n(n+1)}}{(1+q)\left(1+q^{3}\right) \cdots\left(1+q^{2 n+1}\right)}, \\
\rho(q) & =\sum_{n=0}^{\infty} \frac{q^{2 n(n+1)}}{\left(1+q+q^{2}\right)\left(1+q^{3}+q^{6}\right) \cdots\left(1+q^{2 n+1}+q^{4 n+2}\right)} .
\end{aligned}
$$

The functions $\omega, \nu$, and $\rho$ are supplied by Watson [3], who published an account of Ramanujan's letter together with proofs and amplifications of some of the chief properties of the functions asiserted by Ramanujan. In particular, Watson proves the fundamental relations (1.2) connecting the functions (1.1).

$$
\begin{aligned}
& \text { (a) } 2 \phi(-q)-f(q)=f(q)+4 \psi(-q)=\vartheta_{4}(0, q) \prod_{r=1}^{\infty}\left(1+q^{r}\right)^{-1}, \\
& \text { (b) } 4 \chi(q)-f(q)=3 \vartheta_{4}^{2}\left(0, q^{3}\right) \prod_{r=1}^{\infty}\left(1-q^{r}\right)^{-1}, \\
& \text { (c) } 2 \rho(q)+\omega(q)=3\left[\frac{1}{2} q^{-3 / 8} \vartheta_{2}\left(0, q^{3 / 2}\right)\right]^{2} \prod_{r=1}^{\infty}\left(1-q^{2 r}\right)^{-1}, \\
& \text { (d) } \nu( \pm q) \pm q \omega\left(q^{2}\right)=\frac{1}{2} q^{-1 / 4} \vartheta_{2}(0, q) \prod_{r=1}^{\infty}\left(1+q^{2 r}\right) .
\end{aligned}
$$


The classification of third order applied to this set of mock theta functions probably refers to the form of the ordinary theta functions appearing in the right members of these identities. We note that

$$
\vartheta_{4}(0, q) \prod_{r=1}^{\infty}\left(1+q^{r}\right)^{-1}=i q^{-1 / 8} \frac{\vartheta_{4}^{2}(0 / \tau)}{\vartheta_{1}\left(\frac{\tau}{2} / \frac{3 \tau}{2}\right)},
$$

$$
3 \vartheta_{4}^{2}\left(0, q^{3}\right) \prod_{r=1}^{\infty}\left(1-q^{r}\right)^{-1}=3 i q^{-1 / 8} \frac{\vartheta_{4}^{2}(0 / 3 \tau)}{\vartheta_{1}\left(\frac{\tau}{2} / \frac{3 \tau}{2}\right)}
$$

(c)

(d)

$$
\begin{aligned}
\frac{3}{4} q^{-3 / 4} \vartheta_{2}^{2}\left(0, q^{3 / 2}\right) \prod_{r=1}^{\infty}\left(1-q^{2 r}\right)^{-1} & =\frac{3}{4} i q^{-1} \frac{\vartheta_{2}^{2}\left(0 / \frac{3 \tau}{2}\right)}{\vartheta_{1}(\tau / 3 \tau)}, \\
\frac{1}{2} q^{-1 / 4} \vartheta_{2}(0, q) \prod_{r=1}^{\infty}\left(1+q^{2 r}\right) & =\frac{1}{4} i q^{-1} \frac{\vartheta_{2}^{2}(0 / \tau)}{\vartheta_{1}(2 \tau / 6 \tau)}
\end{aligned}
$$

each denominator being of the form $\vartheta_{1}(\alpha / 3 \alpha)$. Analogous remarks hold for the fifth order functions (but apparently not for those of seventh order for which no relations of the type (1.2) are known).

With regard to the characteristic mock theta property, Ramanujan asserts: when $q=-e^{-\pi t}$ and $t \rightarrow 0+$,

$$
f(q)+\frac{1}{t^{1 / 2}} e^{(\pi / 24)(1 / t-t)} \rightarrow 4 .
$$

This asymptotic representation is analogous to that quoted above for $P(q)$ and is derived in the same way from the linear transformation theory for $f(q)$ developed by Watson (see (3.1) below). The transformation equation needed here may be written in the form

$$
\begin{aligned}
f\left(-e^{-\pi t}\right)= & \frac{-1}{t^{1 / 2}} e^{(\pi / 24)(1 / t-t)} f\left(-e^{-\pi / t}\right) \\
& +2(6 t)^{1 / 2} e^{-\pi t / 24} \int_{0}^{\infty} e^{-(3 \pi t / 2) x^{2}} \frac{\cosh 5 \pi t x / 2+\cosh \pi t x / 2}{\cosh 3 \pi t x} d x .
\end{aligned}
$$

Here let $t \rightarrow 0$. It is easy to show that the dominant part of the integrand is $2 e^{-(3 \pi t / 2) x^{2}}$, and thence to derive the result (1.3).

Now by an extension of Watson's linear transformation formulae for the functions we shall prove similar asymptotic representations in closed exponential form for $f(q)$ as $q$ approaches any singular rational point of the unit circle. This result is contained in the statement of the following theorem: 
Theorem I. Let $(h, k)=1$, and $h h^{\prime} \equiv-1(\bmod k)$. For all complex $z$ such that $\Re(z)>0,|\Im(z)| \leqq 1 /(k+1)$,

$$
f\left(e^{\pi i(h+i z) / k}\right)=\left\{\begin{array}{c}
\epsilon_{h, k} \frac{1}{z^{1 / 2}} e^{-\pi z / 24 k+\pi z^{-1} / 24 k} f\left( \pm e^{\pi i\left(h^{\prime}+i z^{-1}\right) / k}\right) \\
+O(k \log k),(h \text { odd }) \\
\eta_{h, k} \frac{2^{3 / 2}}{z^{1 / 2}} e^{-\pi z / 24 k-4 \pi z^{-1} / 3 k} \omega\left(e^{2 \pi i\left(h^{\prime}+i z^{-1}\right) / k}\right) \\
+O(k \log k),(h \text { even }),
\end{array}\right.
$$

where

$$
\begin{array}{r}
\epsilon_{h, k}=(-1)^{k+\left(h^{2}-1\right) / 8} \exp \left[\pi i \frac{\left(2 k^{2}+1\right) h^{\prime \prime}\left(h^{2}-1\right) / 2-h\left(k^{2}-1\right)}{12 k}\right] \\
\cdot \frac{1}{2 k^{1 / 2}} \sum_{\mu=0}^{2 k-1} e^{\pi i((h+k) / k) \mu^{2}},(h \text { odd }),
\end{array}
$$

and $h h^{\prime \prime} \equiv-1(\bmod 2 k)$, and $\left|\eta_{h, k}\right|=\left|\epsilon_{h, k}\right|=1$, and $\omega(q)$ is another mock theta function defined by (1.1).

It will be shown that the rational points $e^{\pi i h / k}$ are essential singularities of $f(q)$ when $h$ is odd, and we observe that in this case $\epsilon_{h, k}$ is given explicitly by (1.6). The exponential sum appearing in its definition may be evaluated as a well known Gaussian sum:

$$
\frac{1}{2 k^{1 / 2}} \sum_{\mu=0}^{2 k-1} e^{\pi i((h+k) / k) \mu^{2}}= \begin{cases}\frac{1+i}{2^{1 / 2}} i^{((h+k-1) / 2)^{2}}\left(\frac{-k / 2}{h+k}\right) & (k \text { even }), \\ i^{((k-1) / 2)^{2}}\left(\frac{(h+k) / 2}{k}\right) & (k \text { odd }),\end{cases}
$$

where we employ the Jacobi symbol of quadratic character. The ambiguous sign of argument $\left( \pm e^{\pi i\left(h^{\prime}+i z^{-1}\right) / k}\right)$ in (1.5) is dependent on $h$ and $k$ and can be determined by a certain algorithm, as indicated in the proof of Lemma 4, below. For either choice of sign, as $q \rightarrow e^{\pi i h / k}$ radially (real $\left.z\right), f\left( \pm e^{\pi i\left(h^{\prime}+i z^{-1}\right) / k}\right.$ ) $\rightarrow f(0)=1$, so that, replacing $O(k \log k)$ by $O(1)$ (since we regard $k$ as fixed for the moment), as $z \rightarrow 0+$,

$$
f\left(e^{\pi i(h+i z) / k}\right)-\frac{\epsilon_{h, k}}{z^{1 / 2}} e^{-\pi z / 24 k+\pi z^{-1} / 24 k}=O(1),
$$

the announced analogue of (1.3).

On the other hand, when $h$ is even, $f(q)$ is bounded on radial approach to $e^{\pi i h / k}$. In this case the factor $\eta_{h, k}$ which depends in a complicated (but de- 
terminate) fashion on $h$ and $k$, need not be evaluated beyond the fact that $\left|\eta_{h, k}\right|=1$.

Now with the help of Theorem I we can establish this hitherto unproved assertion of Ramanujan: if $f(q)=\sum_{n=0}^{\infty} A(n) q^{n}$, then

$$
A(n)=(-1)^{n-1} \frac{e^{\pi((n-1 / 24) / 6)^{1 / 2}}}{2(n-1 / 24)^{1 / 2}}+O\left(\frac{e^{(\pi / 2)((n-1 / 24) / 6)^{1 / 2}}}{(n-1 / 24)^{1 / 2}}\right) .
$$

This result arises chiefly from (1.3) by the Hardy-Ramanujan method [2], which utilizes the fact that the function is sharply characterized by the behavior in the neighborhood of its singularities. We expect that $f(q)$ has its "worst" singularity at $q=-1$ where the $m$ th term of the defining sum (1.1) has a pole of order $2[(m+1) / 2]$. Accordingly the equation (1.4) representing the functional behavior near $q=-1$ gives the principal term for $A(n)$ in (1.7).

Moreover, since Theorem I gives expressions analogous to (1.4) for every rational point, we can compute the respective contribution to $A(n)$ of each of these singularities to obtain a refinement of the large error term of exponential order (1.7). The main result of this paper is embodied in Theorem II, which gives the improved asymptotic formula for $A(n)$.

Theorem II. The coefficient of $q^{n}$ in the series $f(q)=\sum A(n) q^{n}$ is

$$
A(n)=\sum_{0<k \leqq n^{1 / 2}} \lambda(k) e^{(\pi / k)((n-1 / 24) / 8)^{1 / 2}}+O\left(n^{1 / 2} \log n\right),
$$

where

$$
\begin{aligned}
& \lambda(1)=(-1)^{n-1} / 2, \\
& \lambda(k)=\frac{1}{2}\left\{\sum_{(h, 2 k)=1,-k<h<k} e^{x i(h / k) n_{\epsilon_{h, k}}}\right\} \quad\left(k=2,3, \cdots,\left[n^{1 / 2}\right]\right) .
\end{aligned}
$$

By taking the optimum number $\left[n^{1 / 2}\right]$ of terms of the series (1.8) we have been able to obtain an estimate of error quite small in comparison with the exponential size of the terms themselves.

Theorems of the same form as I and II hold for all the functions of third order defined by (1.1).

Whether or not a complete theory of linear transformations exists for the functions of fifth and seventh order is problematical. While the behavior of the fifth order functions on radial approach to the rational points of the natural boundary is known [4], transformation formulae of the type (1.5) are not so easily available owing to the lack of certain identities necessary to our argument (see (3.1) below). The set of functions of seventh order is even more difficult to handle, since there are no known relations of the type (1.2) among these functions.

To Professor H. A. Rademacher I wish to express my gratitude for suggesting this problem to me. His constant encouragement and many helpful 
suggestions have been a source of inspiration throughout the course of this work.

2. Two lemmas. For the proof of Theorem I, we prepare two lemmas.

The mock theta property of $f(q)$ is highlighted by the relation (1.2a) which we may rewrite

$$
f(q)=G(q)-4 \psi(-q)=-G(q)+2 \phi(-q)
$$

if

$$
G(q)=\vartheta_{4}(0, q) \prod_{r=1}^{\infty}\left(1-q^{2 r-1}\right) .
$$

It will be seen that for radial approach to a given rational point $e^{\pi i h / k}$ of the unit circle

$$
\begin{aligned}
f(q)-G(q) & =O(1) & & (h \text { odd, } k \text { even }), \\
f(q)+G(q) & =O(1) & & (h \text { odd, } k \text { odd }),(h, k)=1, \\
f(q) & =O(1) & & (h \text { even }),
\end{aligned}
$$

so that it will be natural to obtain the dominant terms in the asymptotic representation of $f(q)$ near these points from the theta series $G(q)$. To show (2.2) it suffices to prove

Lemma 1. Let $q=\rho e^{\pi i h / k}$, real $\rho>0$, and let $\rho \rightarrow 1-$. Then for fixed $h$ and $k$ such that $(h, k)=1$,

$$
\begin{array}{ll}
f(q)=O(1) & (h \text { even, } k \text { odd }), \\
\phi(q)=O(1) & (h \text { even }, k \text { odd }), \\
\psi(q)=O(1) & (h \text { odd }, k \text { even }) .
\end{array}
$$

The proof is virtually the same for each. For $h$ even,

$$
\begin{aligned}
f(q) & \left.=\sum_{m=0}^{\infty} \frac{q^{m^{2}}}{\prod_{r=1}^{m}\left(1+q^{r}\right)^{2}}=\sum_{m=0}^{\infty} \frac{\rho^{m^{2}} e^{\pi i(h / k) m^{2}}}{\prod_{r=1}^{m}\left(1+\rho^{r} e^{\pi i(h / k) r}\right)^{2}} \quad \text { (put } m=\mu k+\nu\right) \\
& =\sum_{\nu=0}^{k-1} \sum_{\mu=0}^{\infty} \frac{\rho^{(\mu k+\nu)^{2}} e^{\pi i(h / k)(\mu k+\nu)^{2}}}{\prod_{r=1}^{\mu k+\nu}\left(1+\rho^{r} e^{\pi i(h / k) r}\right)^{2}}=\sum_{\nu=0}^{k-1} \sum_{\mu=0}^{\infty} a_{\nu, \mu}, \quad \text { say. }
\end{aligned}
$$

Then for $\nu=0,1, \cdots, k-1$, and $\rho>0, \sum_{\mu} a_{\nu, \mu}$ is uniformly convergent:

$$
\left|\frac{a_{\nu, \mu+1}}{a_{\nu, \mu}}\right|=\frac{\rho^{(\mu k+\nu+k)^{2}-(\mu k+\nu)^{2}}}{\prod_{r=\mu k+\nu+1}^{\mu k+\nu+k}\left|1+\rho^{r} e^{\pi i(h / k) r}\right|^{2}} .
$$


We estimate the denominator, making use of the inequality $\left|1+R e^{i \theta}\right|^{2}$ $\geqq R\left|1+e^{i \theta}\right|^{2}$.

$$
\begin{aligned}
\prod_{r=\mu k+\nu+1}^{\mu k+\nu+k}\left|1+\rho^{r} e^{\pi i(h / k) r}\right|^{2} & =\prod_{r=1}^{k}\left|1+\rho^{r+\mu k+\nu} e^{\pi i(h / k)(r+\nu)}\right|^{2} \\
& \geqq \prod_{r=1}^{k} \rho^{r+\mu k+\nu}\left|1+e^{\pi i(h / k)(r+\nu)}\right|^{2} \\
& =\rho^{k(\mu k+\nu)+k(k+1) / 2} \prod_{r=1}^{k}\left|1+e^{\pi i(h / k)(r+\nu)}\right|^{2} .
\end{aligned}
$$

Now as $r$ runs modulo $k$, so does $r^{\prime}=h(r+\nu) / 2$. Note that $h$ is even, $k$ is odd.

$$
\prod_{r=1}^{k}\left|1+e^{\pi i(h / k)(r+\nu)}\right|^{2}=\prod_{r^{\prime}=1}^{k}\left|1+e^{2 \pi i\left(r^{\prime} / k\right)}\right|^{2}=4 .
$$

Combining (2.6), (2.7), and (2.8), we have

so that

$$
\left|\frac{a_{\nu, \mu+1}}{a_{\nu, \mu}}\right| \leqq \frac{\rho^{(\mu k+\nu) k+k(k-1) / 2}}{4} \leqq \frac{1}{4},
$$

$$
\begin{aligned}
|f(q)| & \leqq \sum_{\nu=0}^{k-1} \sum_{\mu=0}^{\infty} \frac{1}{4^{\mu}}\left|a_{\nu, 0}\right|=\frac{4}{3} \sum_{\nu=0}^{k-1} \frac{\rho^{\nu^{2}}}{\prod_{r=1}^{\nu} \mid 1+\rho^{r} e^{\pi i(h / k) r \mid}} \\
& \leqq \frac{4}{3} \sum_{\nu=0}^{k-1} \frac{1}{\left|1+e^{\pi i(k-1) / k}\right|^{2 \nu}}=O(1) \quad \text { for fixed } k \text { as } \rho \rightarrow 1 .
\end{aligned}
$$

The proof of (2.4) and (2.5) is the same, except that when $k$ is even we use, in place of (2.8),

$$
\prod_{r=1}^{k}\left|1+e^{\pi i(2 r-1) / k}\right|=2 .
$$

Now it is easy to combine the results of Lemma 1 with (2.1) to yield (2.2). For the treatment of $G(q)$ we prepare

Lemma 2. For $h$ odd and $(h, k)=1$, and complex $z, \Re(z)>0$,

$$
G\left(e^{\pi i(h+i z) / k}\right)=(-1)^{k} \epsilon_{h, k} \frac{1}{z^{1 / 2}} e^{-\pi z / 24 k+\pi z^{-1} / 24 k}\left\{1+\sum_{m=1}^{\infty} c_{h, k}(m) e^{-(\pi / k z) m}\right\}
$$
where $\sum c_{h, k}(m) x^{m}$ is convergent for $|x|<1$ and $\epsilon_{h, k}$ is given by (1.6).

Proof. Since $G(q)=\vartheta_{4}(0, q) \prod_{r=1}^{\infty}\left(1-q^{2 r-1}\right)$ we use the well known transformation for the modular form $\eta(\tau)$ to write 


$$
\prod_{r=1}^{\infty}\left(1-e^{\pi i((h+i z) / k)(2 r-1)}\right)=\frac{\prod_{r=1}^{\infty}\left(1-e^{\pi i((h+i z) / k) r}\right)}{\prod_{r=1}^{\infty}\left(1-e^{\pi i((h+i z) / k) 2 r}\right)}
$$

$(2.10)$

$$
\begin{aligned}
& =\frac{\omega_{h, k} z^{1 / 2} e^{\pi / 12 k z-\pi z / 12 k} \prod_{r=1}^{\infty}\left(1-e^{\pi i\left(\left(h^{\prime \prime}+i z^{-1}\right) / k\right) 2 r}\right)^{-1}}{\omega_{h, 2 k} z^{1 / 2} e^{\pi / 24 k z-\pi z / 24 k} \prod_{r=1}^{\infty}\left(1-e^{\pi i\left(\left(h^{\prime \prime}+i z^{-1}\right) / k\right) r}\right)^{-1}} \\
& =\frac{\omega_{h, k}}{\omega_{h, 2 k}} e^{(\pi / 24 k)(1 / z-z)} \prod_{r=1}^{\infty}\left(1-e^{\pi i\left(\left(h^{\prime \prime}+i z^{-1}\right) / k\right)(2 r-1)}\right)
\end{aligned}
$$

where $h h^{\prime \prime} \equiv-1(\bmod 2 k)$, and:

$\omega_{h, k}=\left(\frac{-k}{h}\right) \exp \left[-\pi i\left\{\frac{1}{4}(2-h-h k)\right.\right.$

$$
\left.\left.+\frac{1}{12}\left(k-\frac{1}{k}\right)\left(2 h+h^{2} h^{\prime \prime}-h^{\prime \prime}\right)\right\}\right]
$$

If we use the fact that

$$
\left(\frac{-k}{h}\right)\left(\frac{-2 k}{h}\right)=\left(\frac{2}{h}\right)=(-1)^{\left(h^{2}-1\right) / 8},
$$

$$
\frac{\omega_{h, k}}{\omega_{h, 2 k}}=(-1)^{\left(h^{2}-1\right) / 8} \exp \left[\pi i \frac{h^{\prime \prime}\left(h^{2}-1\right) / 2 \cdot\left(2 k^{2}+1\right)-h\left(k^{2}-1\right)}{12 k}\right] \text {. }
$$

We find the transformation for $\vartheta_{4}(0, q)$ as usual:

$$
\begin{aligned}
\vartheta_{4}^{\prime}(0, q)= & \sum_{m=-\infty}^{\infty}(-1)^{m} e^{\pi i((h+i z) / k) m^{2}} \\
& =\sum_{\nu=0}^{k-1}(-1)^{\nu} e^{\pi i(h / k) \nu^{2}} \frac{1}{(k z)^{1 / 2}} \sum_{\lambda=-\infty}^{\infty} e^{2 \pi i(\nu / k) \lambda-(\pi / k z) \lambda^{2}} \\
& =\frac{1}{(k z)^{1 / 2}}\left\{\sum_{\nu=0}^{k-1} e^{\pi i((h+k) / k) \nu^{2}}\right. \\
& \left.\quad+\sum_{\lambda=1}^{\infty} e^{-(\pi / k z) \lambda^{2}}\left(\sum_{\nu=0}^{k-1} e^{\pi i((h+k) / k) \nu^{2}} 2 \cos \frac{2 \pi \lambda \nu}{k}\right)\right\} \\
& =\frac{1}{(k z)^{1 / 2}} \sum_{\nu=0}^{k-1} e^{\pi i((h+i z) / k) \nu^{2}}\left\{1+\sum_{\lambda=1}^{\infty} c_{1}(\lambda) e^{-(\pi / k z) \lambda^{2}}\right\},
\end{aligned}
$$


making the obvious definition for $C_{1}(\lambda)$. Combining (2.10), (2.11), and (2.12), we write

$$
\begin{aligned}
G\left(e^{\pi i(h+i z) / k}\right) & \\
= & (-1)^{\left(h^{2}-1\right) / 8} \exp \left[\pi i \frac{h^{\prime \prime}\left(h^{2}-1\right) / 2 \cdot\left(2 k^{2}+1\right)-h\left(k^{2}-1\right)}{12 k}\right] e^{(\pi / 24 k)(1 / z-z)} \\
& \cdot \prod_{r=1}^{\infty}\left(1-e^{\pi i\left(\left(h^{\prime \prime}+i z^{-1}\right) / k\right)(2 r-1)}\right) \cdot \sum_{\nu=0}^{k-1} e^{\pi i((h+k) / k) \nu^{2}}\left\{1+\sum_{\lambda=1}^{\infty} c_{1}(\lambda) e^{-(\pi / k z) \lambda^{2}}\right\} .
\end{aligned}
$$

For simplicity we consider the infinite product as identical with a certain power series

$$
\prod_{r=1}^{\infty}\left(1-\left[e^{\pi i h^{\prime \prime} / k-\pi / k z}\right]^{2 r-1}\right)=1+\sum_{r=1}^{\infty} c_{2}(r) e^{-(\pi / k z) r},
$$

where this series must converge for $\left|e^{-\pi / k z}\right|<1$.

Then using the definition of $\epsilon_{h, k}(1.6)$,

$$
\begin{aligned}
G\left(e^{\pi i(h+i z) / k}\right)= & (-1)^{k} \epsilon_{h, k} \frac{e^{(\pi / 24 k)(1 / z-z)}}{z^{1 / 2}}\left\{1+\sum_{r=1}^{\infty} c_{2}(r) e^{-(\pi / k z) r}\right\} \\
& \cdot\left\{1+\sum_{\lambda=0}^{\infty} c_{1}(\lambda) e^{-(\pi / k z) \lambda^{2}}\right\} .
\end{aligned}
$$

On multiplying the power series we have the desired lemma.

3. Transformation formulae. We turn now to the linear transformation formulae for the function $f(q)$. Basic equations of modular type have been proved by Watson [3] expressing each of the ten functions $f, \phi, \psi, \nu, \omega\left( \pm e^{\pi i \tau}\right)$ in terms of mock theta functions of $\left( \pm e^{-\pi i / r}\right)$. In particular we quote here the three formulae:

$$
\begin{aligned}
e^{-\pi i \tau / 24} f\left(e^{\pi i \tau}\right) & =\frac{2^{3 / 2}}{(-i \tau)^{1 / 2}} e^{-4 \pi i / 3 \tau} \omega\left(e^{-2 \pi i / \tau}\right)+\frac{4\left(3^{1 / 2}\right) i}{\tau} J_{2}\left(\frac{\pi i}{\tau}\right), \\
e^{-\pi i \tau / 24} f\left(-e^{\pi i \tau}\right) & =\frac{-1}{(-i \tau)^{1 / 2}} e^{\pi i / 24 \tau} f\left(-e^{-\pi i / \tau}\right)+\frac{2\left(6^{1 / 2}\right) i}{\tau} J_{0}\left(\frac{\pi i}{\tau}\right), \\
e^{4 \pi i \tau / 3} \omega\left(e^{2 \pi i \tau}\right) & =\frac{1}{2^{3 / 2}} \frac{1}{(-i \tau)^{1 / 2}} e^{\pi i / 24 \tau} f\left(e^{-\pi i / \tau}\right)-\frac{3^{1 / 2} i}{\tau} J_{1}\left(\frac{\pi i}{\tau}\right),
\end{aligned}
$$

where $\omega(q)$ is another mock theta function (see (1.1)) and

$$
J_{\nu}(\alpha)=\int_{0}^{\infty} e^{-3 \alpha x^{2} / 2} H_{\nu}(\alpha x) d x \quad(\nu=0,1,2) .
$$

The $H_{\nu}(\alpha x)$ are defined in Lemma 3 below. The terms involving $J_{\nu}(\alpha)$ are 
in the nature of error terms which we shall show to be bounded for all $\Re(\alpha)>0$.

Lemma 3. If

$$
J_{\nu}(\alpha)=\int_{0}^{\infty} e^{-3 \alpha x^{2} / 2} H_{\nu}(\alpha x) d x
$$

where

$$
\begin{gathered}
H_{0}(\alpha x)=\frac{\cosh \alpha x / 2+\cosh 5 \alpha x / 2}{\cosh 3 \alpha x}, \\
H_{1}(\alpha x)=\frac{\sinh \alpha x}{\sinh 3 \alpha x / 2}, H_{2}(\alpha x)=\frac{\cosh \alpha x}{\cosh 3 \alpha x},
\end{gathered}
$$

then there exists $K>0$ such that $\left|\alpha J_{\nu}(\alpha)\right|<K$ for all $\Re(\alpha)>0$, i.e., $\alpha J_{\nu}(\alpha)$ is uniformly bounded in this half-plane.

Proof. If $z=x+i y(x>0)$, then $\left|H_{\nu}(z)\right|<e^{-x / 3}$ for large $x$. Now suppose $\alpha=C e^{i A}$ where $|A|<\pi / 2$, and $C>0$. Make the substitution $z=\alpha x$ :

$$
J_{\nu}(\alpha)=\frac{1}{\alpha} \int_{S} e^{-(3 / 2)(1 / \alpha) z^{2}} H_{\nu}(z) d z
$$

where $z$ runs on the ray $S$ from 0 through $\alpha(\operatorname{Arg} z=A)$. But we can shift the path of integration from $S$ to the positive real axis, since

$$
\frac{1}{\alpha} \int_{R}^{R e^{i A}} e^{-(3 / 2)(1 / \alpha) z^{2}} H_{\nu}(z) d z \rightarrow 0 \quad \text { as } \quad R \rightarrow \infty
$$

(integral taken along a circular arc of radius $R, z=R e^{i \theta}, 0<|\theta|<A<\pi / 2$ ). Then, since the integrand is pole-free on $\Re(z)>0$,

$$
J_{\nu}(\alpha)=\frac{1}{\alpha} \int_{0}^{\infty} e^{-(3 / 2)(1 / \alpha) x^{2}} H_{\nu}(x) d x \quad \quad(x \text { real }) .
$$

But since $\Re(\alpha)>0$, we have $\left|e^{-(3 / 2)(1 / \alpha) x^{2}}\right|<1$;

$$
\left|\alpha J_{\nu}(\alpha)\right|<\int_{0}^{\infty}\left|H_{\nu}(x)\right| d x<K_{1} \int_{0}^{\infty} e^{-x / 3} d x=K .
$$

This completes the proof of the lemma.

We rewrite the equations (3.1) by defining:

$$
\begin{aligned}
F_{1}(\tau) & =2^{-1} e^{-\pi i \tau / 24} f\left(e^{\pi i \tau}\right), \\
F_{2}(\tau) & =2^{-1} e^{-\pi i \tau / 24} f\left(-e^{\pi i \tau}\right), \\
\Omega(\tau) & =2^{1 / 2} e^{4 \pi i \tau / 3} \omega\left(e^{2 \pi i \tau}\right) .
\end{aligned}
$$


Consider the effect on these functions of a modular transformation of the argument. Let $S \tau=\tau+1$ and $T \tau=-1 / \tau$. We have directly from their definition:

$$
\begin{aligned}
F_{1}(\tau) & =e^{\pi i / 24} F_{2}(S \tau)=e^{\pi i / 12} F_{1}\left(S^{2} \tau\right), \\
\Omega(\tau) & =e^{-4 \pi i / 3} \Omega(S \tau)
\end{aligned}
$$

and from (3.1), with the help of Lemma 3,

$$
\begin{gathered}
F_{1}(\tau)=\frac{1}{(-i \tau)^{1 / 2}} \Omega(T \tau)+O(1), \\
\Omega(\tau)=\frac{1}{(-i \tau)^{1 / 2}} F_{1}(T \tau)+O(1), \\
F_{2}(\tau)=\frac{-1}{(-i \tau)^{1 / 2}} F_{2}(T \tau)+O(1) .
\end{gathered}
$$

As the next step in the proof of Theorem I, we prove Lemma 4, which follows naturally from the quasi-modular equations (3.3), (3.4).

Lemma 4. Let $\tau=(h+i z) / k$ and $\tau^{\prime}=\left(h^{\prime}+i z^{-1}\right) / k$ with $h h^{\prime} \equiv-1(\bmod k)$, $\left|h^{\prime}\right|<k,(h, k)=1$, and suppose $|\Im(\tau)| \leqq 1 /(k+1)$. Then $F_{1}(\tau)$ has either one or another of the representations:

$$
F_{1}(\tau)=\frac{\zeta}{(k \tau-h)^{1 / 2}}\left\{\begin{array}{c}
F_{1}\left(\tau^{\prime}\right) \\
F_{2}\left(\tau^{\prime}\right) \\
\Omega\left(\tau^{\prime}\right)
\end{array}\right\}+O(k \log k),
$$

where $|\zeta|=1$.

Proof. First,

$$
\tau^{\prime}=\frac{h^{\prime} \tau-\left(h h^{\prime}+1\right) / k}{k \tau-h} .
$$

Since this is a modular substitution, it can be analyzed by a continued fraction decomposition into a product of the elementary substitutions $S \tau=\tau+1$ and $T \tau=-1 / \tau$.

$$
\left(\begin{array}{cc}
h^{\prime} & -\left(h h^{\prime}+1\right) / k \\
k & -h
\end{array}\right)=\left(\begin{array}{ll}
l & b \\
c & d
\end{array}\right)=S^{d_{0}} T S^{d_{1}} T \cdots S^{d_{p}}
$$

the substitutions to be applied successively beginning with $S^{d_{p}}$ and ending with $S^{a_{0}}$. The intermediate stages are: 


$$
\begin{aligned}
& \left(\begin{array}{ll}
c & d \\
a_{1} & b_{1}
\end{array}\right)=S^{d_{1}} T S^{d_{2}} T \cdots T S^{d_{p}} \\
& \left(\begin{array}{ll}
a_{1} & b_{1} \\
a_{2} & b_{2}
\end{array}\right)=S^{d_{2}} T \cdots T S^{d_{p}}
\end{aligned}
$$

$$
\begin{aligned}
& \left(\begin{array}{ll}
a_{p-2} & b_{p-2} \\
a_{p-1} & b_{p-1}
\end{array}\right)=S^{d_{p-1}} T S^{d_{p}}, \\
& \left(\begin{array}{ll}
a_{p-1} & b_{p-1} \\
0 & b_{p}
\end{array}\right)=S^{d_{p}},
\end{aligned}
$$

where the $a_{\nu}, b_{\nu}$, and $d_{\nu}$ are integers determined from $a, b, c, d$, by the well known division algorithm

$$
\begin{array}{r}
a \tau+b=d_{0}(c \tau+d)-\left(a_{1} \tau+b_{1}\right), \\
c \tau+b=d_{1}\left(a_{1} \tau+b_{1}\right)-\left(a_{2} \tau+b_{2}\right), \\
\cdot . \cdot \cdot \cdot \cdot \cdot \cdot \cdot \cdot \cdot \cdot \cdot \cdot \cdot \cdot \cdot, \\
a_{p-2} \tau+b_{p-2}=d_{p-1}\left(a_{p-1} \tau+b_{p-1}\right)-b_{p} .
\end{array}
$$

Certain properties are pertinent:

$$
\begin{gathered}
\left|\begin{array}{ll}
a_{\nu-1} & b_{\nu-1} \\
a_{\nu} & b_{\nu}
\end{array}\right|=1, \text { so } \quad\left(a_{\nu}, b_{\nu}\right)=1 \quad(\nu=1, \cdots, p), \\
b_{p}=a_{p-1}= \pm 1 .
\end{gathered}
$$

The $a_{\nu}$ form a decreasing sequence, $|c|>\left|a_{1}\right|>\left|a_{2}\right|>\cdots>\left|a_{p-1}\right|=1$. This implies $|c| \geqq\left|a_{\nu}\right|+\nu(\nu=1, \cdots, p-1)$, in particular for $\nu=p-1,|c| \geqq p$. However we can improve the latter inequality estimating the number of stages $p$ : from the division scheme we see that

$$
\begin{aligned}
a & =d_{0} c-a_{1}, \\
c & =d_{1} a_{1}-a_{2}, \\
a_{1} & =d_{2} a_{2}-a_{3}, \\
\cdot \cdot \cdot \cdot \cdot \cdot \cdot, & \cdot \cdot \\
a_{\nu-1} & =d_{\nu} a_{\nu}-a_{\nu+1},
\end{aligned}
$$

We may perform the division in such a way that

$$
\operatorname{sign}\left(-a_{\nu+1}\right)=\operatorname{sign}\left(d_{\nu} a_{\nu}\right)=\operatorname{sign}\left(a_{\nu-1}\right) .
$$

Then 
(a)

$$
\left|a_{\nu+1}\right|=\left|d_{\nu}\right|\left|a_{\nu}\right|+\left|a_{\nu+1}\right| \geqq\left|a_{\nu}\right|+\left|a_{\nu+1}\right| .
$$

If we compare the decreasing sequence of integers

$$
k=|c|,\left|a_{1}\right|,\left|a_{2}\right|, \cdots,\left|a_{p-1}\right|=1
$$

with the first $p$ Fibonacci numbers

$$
\mathcal{F}(p), \mathcal{F}(p-1), \mathcal{F}(p-2), \cdots, \mathcal{F}(1)
$$

for which

$$
\mathcal{F}(r)=\mathcal{F}(r-1)+\mathcal{F}(r-2)=\frac{1}{5^{1 / 2}}\left[\left(\frac{1+5^{1 / 2}}{2}\right)^{r}-\left(\frac{1-5^{1 / 2}}{2}\right)^{r}\right],
$$

equation (a) makes it clear that $\left|a_{\nu}\right| \geqq \mathcal{F}(p-\nu)$ and in particular

$$
|c| \geqq \mathcal{F}(p) \geqq \frac{1}{5^{1 / 2}}\left(\frac{5^{1 / 2}}{2}\right)^{p-1}
$$

so that immediately,

$$
p=O(\log |c|)=O(\log k) .
$$

In our case, $d_{0}=0$ since $\left|h^{\prime}\right|<k$.

Now we build up $\tau^{\prime}=(a \tau+b) /(c \tau+d)$ stepwise, using (3.3) and (3.4). For the first stage,

$$
\begin{aligned}
& F_{1}(\tau)=e^{(\pi i / 24) d_{p}} \begin{cases}F_{2}\left(S^{d_{p}} \tau\right) & \text { if } d_{p} \text { is odd, } \\
F_{1}\left(S^{d_{p}} \tau\right) & \text { if } d_{p} \text { is even }\end{cases} \\
& =\frac{ \pm e^{(\pi i / 24) d_{p}}}{\left(-i S^{d_{p}}\right)^{1 / 2}}\left\{\begin{array}{c}
F_{2}\left(T S^{d_{p}} \tau\right) \\
\Omega\left(T S^{d_{p}}\right)
\end{array}\right\}+O(1) \\
& =\frac{ \pm e^{(\pi i / 24) d_{p}}}{\left( \pm i\left(a_{p-1} \tau+b_{p-1}\right)\right)^{1 / 2}}\left\{\begin{array}{l}
F_{2}\left(T \frac{a_{p-1} \tau+b_{p-1}}{b_{p}}\right) \\
\Omega\left(T \frac{a_{p-1} \tau+b_{p-1}}{b_{p}}\right)
\end{array}\right\}+O(1) .
\end{aligned}
$$

We observe that the ambiguity of Lemma 4 arises from equation (3.3) and that the correct choice depends on the parity of the powers $d_{1}, d_{2}, \cdots, d_{p}$. We proceed inductively. Assume we have found

$$
\begin{array}{r}
F_{1}(\tau)=\frac{\zeta}{\left(a_{\nu} \tau+b_{\nu}\right)^{1 / 2}}\left\{\begin{array}{c}
F_{1} \\
F_{2} \\
\Omega
\end{array}\right\}\left(T \frac{a_{\nu} \tau+b_{\nu}}{a_{\nu+1} \tau+b_{\nu+1}}\right)+O((p-\nu) k) \\
(\nu=1, \cdots, p-1 ;|\zeta|=1) ;
\end{array}
$$


we can deduce the case for $\nu-1$.

$$
\begin{aligned}
& F_{1}(\tau)=\frac{\zeta}{\left(a_{\nu} \tau+b_{\nu}\right)^{1 / 2}}\left\{\begin{array}{r}
e^{(\pi i / 24) d_{\nu}}\left(\begin{array}{c}
F_{1} \\
F_{2}
\end{array}\right)\left(S^{d_{\nu}} T \frac{a_{\nu} \tau+b_{\nu}}{a_{\nu+1} \tau+b_{\nu+1}}\right) \\
e^{-(4 \pi i / 3) d_{\nu} \Omega}\left(S^{d_{\nu}} T \frac{a_{\nu} \tau+b_{\nu}}{a_{\nu+1} \tau+b_{\nu+1}}\right)
\end{array}\right\} \\
& +O((p-\nu) k) \\
& =\frac{\zeta^{\prime}}{\left(a_{\nu} \tau+b_{\nu}\right)^{1 / 2}}\left[\frac{1}{\left(-i\left(S^{d_{\nu}} T \frac{a_{\nu} \tau+b_{\nu}}{a_{\nu+1} \tau+b_{\nu+1}}\right)\right)^{1 / 2}}\right. \\
& \left.\cdot\left(\begin{array}{c}
\Omega \\
F_{2} \\
F_{1}
\end{array}\right)\left(T S^{d_{\nu}} T \frac{a_{\nu} \tau+b_{\nu}}{a_{\nu+1} \tau+b_{\nu+1}}\right)+O(1)\right]+O((p-\nu) k)
\end{aligned}
$$

by (3.4). Using the relations (3.6) this becomes

$$
\begin{aligned}
F_{1}(\tau)= & \frac{\zeta^{\prime}}{\left(a_{\nu} \tau+b_{\nu}\right)^{1 / 2}}\left[\frac{1}{\left(-i \frac{a_{\nu-1} \tau+b_{\nu-1}}{a_{\nu} \tau+b_{\nu}}\right)^{1 / 2}}\left(\begin{array}{c}
\Omega \\
F_{2} \\
F_{1}
\end{array}\right)\left(T \frac{a_{\nu-1} \tau+b_{\nu-1}}{a_{\nu} \tau+b_{\nu}}\right)\right] \\
& +O\left(\left|a_{\nu} \tau+b_{\nu}\right|^{-1 / 2}\right)+O((p-\nu) k) .
\end{aligned}
$$

We examine the error term

$$
\begin{aligned}
\left|a_{\nu} \tau+b_{\nu}\right|^{1 / 2} & =\left|a_{\nu} \frac{h+i z}{k}+b_{\nu}\right|^{1 / 2}=\left|\frac{a_{\nu} h+b_{\nu} k-a_{\nu} \Im(z)}{k}+i \frac{a_{\nu} \Re(z)}{k}\right|^{1 / 2} \\
& \geqq\left|\frac{a_{\nu} h+b_{\nu} k-a_{\nu} \Im(z)}{k}\right|^{1 / 2} .
\end{aligned}
$$

Now $|\Im(z)| \leqq 1 /(k+1)$ by hypothesis, and $\left|a_{\nu}\right| \leqq k-\nu$. Moreover $\left|a_{\nu} h+b_{\nu} k\right|$ $\geqq 1$; for $a_{\nu} h+b_{\nu} k=0$ would imply $a_{\nu} / b_{\nu}=-k / h$, but $\left(a_{\nu}, b_{\nu}\right)=1$ and $(k,-h)$ $=1$ and $k \geqq\left|a_{\nu}\right|+\nu$ leads to a contradiction. Hence

$$
\begin{gathered}
\left|a_{\nu} \tau+b_{\nu}\right|^{1 / 2} \geqq\left|\frac{1-(k-\nu) /(k+1)}{k}\right|^{1 / 2} \geqq \frac{\text { constant }}{k}, \\
O\left(\left|a_{\nu} \tau+b_{\nu}\right|^{-1 / 2}\right)+O((p-\nu) k)=O(k)+O((p-\nu) k)=O((p-\nu+1) k) . \\
F_{1}(\tau)=\frac{\zeta^{\prime \prime}}{\left(a_{\nu-1} \tau+b_{\nu-1}\right)^{1 / 2}}\left[\left(\begin{array}{c}
\Omega \\
F_{2} \\
F_{1}
\end{array}\right)\left(T \frac{a_{\nu-1} \tau+b_{\nu-1}}{a_{\nu} \tau+b_{\nu}}\right)\right]+O((p-\nu+1) k) .
\end{gathered}
$$


The induction may be continued down to $\nu=1$, giving finally

$$
F_{1}(\tau)=\frac{\zeta}{(c \tau+d)^{1 / 2}}\left[\left(\begin{array}{c}
\Omega \\
F_{2} \\
F_{1}
\end{array}\right)\left(\frac{a \tau+b}{c \tau+d}\right)\right]+O(k \log k) \quad \text { since } p=O(\log k) .
$$

This is equivalent to (3.5) and the lemma is proved.

By applying the definitions (3.2) to the result of this lemma, we find that either one or the other of the following forms prevails for given $h, k$ :

$$
f\left(e^{\pi i(h+i z) / k}\right)=\left\{\begin{aligned}
\frac{\zeta h, k}{z^{1 / 2}} e^{(\pi / 24 k)\left(-z+z^{-1}\right)} f\left( \pm e^{\pi i\left(h^{\prime}+i z^{-1}\right) / k}\right) & \\
& =U_{h, k}(z)+O(k \log k), \\
\frac{\eta_{h, k}}{z^{1 / 2}} 2^{3 / 2} e^{-\pi z / 24 k-4 \pi z^{-1} / 3 k} \omega\left(e^{2 \pi i\left(h^{\prime}+i z^{-1}\right) / k}\right) & +O(k \log k) \\
& =V_{h, k}(z)+O(k \log k)
\end{aligned}\right.
$$

say, where $\left|\zeta_{h, k}\right|=\left|\eta_{h, k}\right|=1$.

It is significant that for real $z \rightarrow 0+$,

$$
\begin{aligned}
\lim _{z \rightarrow 0}\left|U_{h, k}(z)\right| & =\lim _{z \rightarrow 0} \frac{1}{z^{1 / 2}} e^{-\pi z / 24 k+\pi / 24 k z}\left|f\left( \pm e^{\pi i\left(h^{\prime} / k\right)} e^{-\pi / k z}\right)\right| \\
& =\lim _{z \rightarrow 0} \frac{e^{\pi / 24 k z}}{z^{1 / 2}}=\infty,
\end{aligned}
$$

since $f(q)$ is continuous and $f(0)=1$. On the other hand,

$$
\lim _{z \rightarrow 0}\left|V_{h, k}(z)\right|=\lim _{z \rightarrow 0} \frac{2^{3 / 2}}{z^{1 / 2}} e^{-\pi z / 24 k-4 \pi / 3 k z}\left|\omega\left(e^{2 \pi i\left(h^{\prime}+i z^{-1}\right) / k}\right)\right|=0 .
$$

On consulting (2.2) and Lemma 2, we find that for $z$ real, $\left|f\left( \pm e^{\pi i(h+i z) / k}\right)\right|$ $\rightarrow \infty$ when $h$ is odd (the dominant part being $G(q)$ ), but remains bounded when $h$ is even. We conclude

$$
f\left(e^{\pi i(h+i z) / k}\right)=\left\{\begin{array}{lr}
U_{h, k}(z)+O(k \log k), & h \text { odd, } \\
V_{h, k}(z)+O(k \log k), & h \text { even. }
\end{array}\right.
$$

In case $h$ is odd, as $z \rightarrow 0+(k$ fixed),

$$
\begin{aligned}
f(q)-(-1)^{k} G(q)=\frac{1}{z^{1 / 2}} e^{-\pi z / 24 k+\pi z^{-1} / 24 k} & \left\{\zeta_{h, k}\left(1+\sum_{m=1}^{\infty} A(m) q^{m}\right)\right. \\
& \left.-\epsilon_{h, k}\left(1+\sum_{m=1}^{\infty} c_{h, k}(m) e^{-(\pi / k z) m}\right)\right\}+O(1)
\end{aligned}
$$




$$
\begin{aligned}
& =\frac{1}{z^{1 / 2}} e^{-\pi z / 24 k+\pi z^{-1} / 24 k}\left(\zeta_{h, k}-\epsilon_{h, k}\right)+o(1)+O(1) \\
& =O(1)
\end{aligned}
$$

according to (2.2), whence $\zeta_{h, k}=\epsilon_{h, k}$ as defined by (1.6).

This completes the proof of Theorem I. Our method does not give a general expression for $\eta_{h, k}$; but the present statement of the theorem gives exactly the infromation about $f(q)$ needed to prove Theorem II.

4. Proof of Theorem II. The Farey arcs. We follow the Hardy-Ramanujan method [2]. If $f(q)=\sum A(n) q^{n}$, by Cauchy's theorem,

$$
A(n)=\frac{1}{2 \pi i} \int_{C} \frac{f(x)}{x^{n+1}} d x
$$

where $C$ is a circle in the $x$-plane about the origin, of radius $e^{-\pi / n}$. If we put $x=e^{-\pi / n+\pi i \phi^{\prime}}$

$$
A(n)=\frac{1}{2} \int_{-1}^{1} \frac{f\left(e^{-\pi / n+\pi i \phi^{\prime}}\right)}{\left(e^{-\pi / n+\pi i \phi^{\prime}}\right)^{n}} d \phi^{\prime} .
$$

It will be convenient to distinguish between the upper $\left(\phi^{\prime} \geqq 0\right)$ and lower $\left(\phi^{\prime} \leqq 0\right)$ semi-circles of $C$.

The Farey arcs of order $N$ are defined as follows: For every fraction $h / k$ in the Farey series of order $N(0 \leqq h<k \leqq N,(h, k)=1)$ there corresponds an $\operatorname{arc}\left(h / k-\vartheta_{h, k}^{\prime}, h / k+\vartheta_{h, k}^{\prime \prime}\right)$ with

$$
h / k-\vartheta_{h, k}^{\prime}=\frac{h+h_{1}}{k+k_{1}}, \quad h / k+\vartheta_{h, k}^{\prime \prime}=\frac{h+h_{2}}{k+k_{2}}
$$

where $h_{1} / k_{1}$ and $h_{2} / k_{2}$ are the fractions adjacent to $h / k$ in the Farey series of order $N$, and the end points of the arc given by (4.1) are the "mediants" lying between $h / k$ and the adjacent fraction. Using the fact that $\left(h k_{1}-k h_{1}\right)$ $=\left(h_{2} k-k_{2} h\right)=1$, we find

$$
\vartheta_{h, k}^{\prime}=\frac{1}{k\left(k+k_{1}\right)}, \quad \vartheta_{h, k}^{\prime \prime}=\frac{1}{k\left(k+k_{2}\right)}
$$

and since the mediants are in a Farey series of order $>N$, we know

$$
\max \left\{\frac{1}{k\left(k+k_{1}\right)}, \frac{1}{k\left(k+k_{2}\right)}\right\} \leqq \frac{1}{k(N+1)} .
$$

The integration along the upper semi-circle $C\left(0 \leqq \phi^{\prime} \leqq 1\right)$ may be expressed as the sum of integrals along these Farey arcs $\left(-\vartheta_{h, k}^{\prime} \leqq \phi \leqq \vartheta_{h, k}^{\prime \prime}\right)$ where $\phi=\phi^{\prime}-h / k$ for $0<h<k<N$ and $(h, k)=1$.

The lower semi-circle $\left(-1 \leqq \phi^{\prime} \leqq 0\right)$ containing the fractions $\phi^{\prime}=h / k$ 
with $0 \leqq|-h|<k<N$ may be broken up in an exactly symmetrical manner. We suppose that the point $\phi^{\prime}=0 / 1$ is covered by the arc $-1 /(N+1) \leqq \phi^{\prime}$ $\leqq 1 /(N+1)$ and (exceptionally) $\phi^{\prime}=1 / 1$ by $N /(N+1) \leqq \phi^{\prime} \leqq(N+2) /(N+1)$, i.e., $-1 /(N+1) \leqq \phi \leqq 1 /(N+1)$.

$$
A(n)=\frac{1}{2} \sum_{h, k} \int_{-\vartheta_{h, k}^{\prime}}^{\vartheta_{h, k}^{\prime \prime}} \frac{f\left(e^{-\pi / n+\pi i h / k+\pi i \phi}\right)}{\left(e^{-\pi / n+\pi i h / k+\pi i \phi}\right)^{n}} d \phi
$$

where the summation runs over all $h, k$ such that $0 \leqq|h| \leqq k \leqq N,(h, k)=1$ but $h+k \neq 0$. The choice of $N$ is at our disposal.

In (4.4) we put

$$
A(n)=\frac{1}{2} \sum_{h, k} e^{-\pi i(h / k) n} \int_{-\vartheta_{h, k}^{\prime}}^{\vartheta_{h, k}^{\prime \prime}} f\left(e^{\pi i(h / k)-\pi z / k}\right) e^{(\pi z / k) n} d \phi .
$$

Then, by (4.3), $|\Im(z)| \leqq 1(N+1) \leqq 1 /(k+1)$, satisfying the hypothesis of Theorem I. Accordingly we use the representations (1.4) for $f(q)$.

$$
\begin{aligned}
A(n)= & \frac{1}{2} \sum_{1} e^{-\pi i(h / k) n} \epsilon_{h, k} \\
& \cdot \int_{-\vartheta_{h, k}^{\prime}}^{\vartheta^{\prime \prime}{ }_{h, k}} e^{\pi z n / k}\left\{\frac{1}{z^{1 / 2}} e^{-\pi z / 24 k+\pi z^{-1 / 24 k}} f\left( \pm e^{\pi i\left(h^{\prime}+i z^{-1}\right) / k}\right)+\theta_{1}^{(k)}\right\} d \phi \\
& +\frac{1}{2} \sum_{2} e^{-\pi i(h / k) n}\left(2^{3 / 2}\right) \eta_{h, k} \\
& \cdot \int_{-\vartheta_{h, k}^{\prime}}^{\vartheta^{\prime \prime}{ }_{h, k}} e^{\pi z n / k}\left\{\frac{1}{z^{1 / 2}} e^{-\pi z / 24 k-4 \pi z^{-1} / 3 k} \omega\left(e^{2 \pi i\left(h^{\prime}+i z^{-1}\right) / k}\right)+\theta_{2}^{(k)}\right\} d \phi \\
= & \frac{1}{2}\left\{\sum_{1}+\sum_{2}\right\},
\end{aligned}
$$

say, where $\left|\theta_{i}^{(k)}\right|<C_{1} k \log k(i=1,2)$; and $\sum_{1}$ implies summation over $h, k$ with $h$ odd, $0 \leqq|h| \leqq k \leqq N,(h, k)=1, h+k \neq 0$; and $\sum_{2}$ implies summation over corresponding $h, k$ with $h$ even.

The rational points appearing in $\sum_{2}$ are of the type for which $f(q)$ is bounded on radial approach, hence the associated contributions to $A(n)$ are smaller than those appearing in $\sum_{1}$. For the estimation of $\sum_{2}$ it is advantageous to choose $N=\left[n^{1 / 2}\right]$, so that $\Re(z)=k / n$;

$$
\Re\left(z^{-1}\right)=\frac{k / n}{k^{2} / n^{2}+k^{2} \phi^{2}}>\frac{k / n}{k^{2} / n^{2}+1 / N^{2}}>\frac{k}{2} .
$$

Referring to the definition (1.1) we observe that if $\omega(q)=\sum_{m=0}^{\infty} B(m) q^{m}$ for $|q|<1$, then $B(m) \geqq 0$ for all $m$, and 


$$
\left|\omega\left(e^{2 \pi i\left(h^{\prime}+i z^{-1}\right) / k}\right)\right| \leqq \sum_{m=0}^{\infty} B(m) e^{-(2 \pi / k) \Re\left(z^{-1}\right) m}<\omega\left(e^{-\pi}\right) .
$$

Then

$$
\begin{aligned}
\left|\sum_{2}\right|< & \sum_{2}\left(2^{3 / 2}\right) \\
& \cdot \int_{-\vartheta_{h, k}^{\prime}}^{\vartheta^{\prime \prime}{ }_{h, k}} e^{(\pi n / k) \Re(z)}\left\{\frac{e^{-(\pi / 24 k) \Re(z)-(4 \pi / 3 k) \Re\left(z^{-1}\right)}}{(k / n)^{1 / 2}} \omega\left(e^{-\pi}\right)+C_{1} k \log k\right\} d \phi .
\end{aligned}
$$

The path length $\vartheta_{h, k}^{\prime \prime}+\vartheta_{h, k}^{\prime}<2 / k(N+1)<2 / k n^{1 / 2}$, by (4.3). Using (4.6) and (4.7),

$$
\left|\sum_{2}\right|<C \sum_{h, k} \frac{1}{k n^{1 / 2}}\left\{\frac{1}{(k / n)^{1 / 2}}+k \log k\right\}<C n^{1 / 2} \log n .
$$

With this result, (4.5) becomes

$$
\begin{aligned}
A(n)= & \frac{1}{2} \sum_{1} e^{-\pi i(h / k) n} \epsilon_{h, k} \int_{-\vartheta_{h, k}^{\prime}}^{\vartheta_{h, k}^{\prime \prime}} \frac{e^{(\pi z / k)(n-1 / 24)+\pi z^{-1} / 24 k}}{z^{1 / 2}} d \phi \\
& +\frac{1}{2} \sum_{1} e^{-\pi i(h / k) n} \epsilon_{h, k} \\
& \cdot \int_{-\vartheta_{h, k}^{\prime}}^{\vartheta^{\prime \prime}{ }_{h, k}}\left[e^{(\pi z / k)(n-1 / 24)+\pi z^{-1 / 24 k}}\left\{f\left( \pm e^{\pi i\left(h^{\prime}+i z^{-1}\right) / k}\right)-1\right\}+e^{(\pi z / k) n} \theta_{1}(k)\right] d \phi \\
& +O\left(n^{1 / 2} \log n\right) \\
= & \frac{1}{2}\left\{S_{1}+S_{2}\right\}+O\left(n^{1 / 2} \log n\right),
\end{aligned}
$$

Again (4.6) affords an estimation of $S_{2}$, for

$$
\begin{aligned}
\left|e^{\pi z^{-1} / 24 k}\left(f\left( \pm e^{\pi i\left(h^{\prime}+i z^{-1}\right) / k}\right)-1\right)\right| & \leqq \sum_{m=1}^{\infty}|A(m)| e^{-(\pi / k) \Re\left(z^{-1}\right)(m-1 / 24)} \\
& <\sum_{m=1}^{\infty}|A(m)| e^{-(\pi / 2)(m-1 / 24)}<C_{2} .
\end{aligned}
$$

Also $\left|\theta_{1}^{(k)}\right|<C_{1} k \log k$, so (as in (4.8))

$$
\begin{aligned}
\left|S_{2}\right| & <C \sum_{h, k} \frac{1}{k n^{1 / 2}}\left[\frac{C_{2}}{(k / n)^{1 / 2}}+C_{1} k \log k\right]<C n^{1 / 2} \log n . \\
A(n) & =\frac{1}{2} \sum_{1} e^{-\pi i(h / k) \epsilon_{h, k}} \int_{-\vartheta_{h, k}^{\prime}}^{\vartheta^{\prime \prime}{ }_{h, k}} \frac{e^{(\pi z / k)(n-1 / 24)+\pi z^{-1} / 24 k}}{z^{1 / 2}} d \phi+O\left(n^{1 / 2} \log n\right) \\
& =\frac{1}{2} \sum_{1} e^{-\pi i(h / k) n_{\epsilon_{h, k}} I_{h, k}}+O\left(n^{1 / 2} \log n\right) .
\end{aligned}
$$


5. Evaluation of an integral. Before evaluating the integrals appearing in (4.9) we extend the path of integration so as to symmetrize. Since $1 / k\left(k+k_{i}\right)<1 / k N$

$$
\int_{-\vartheta_{h, k}^{\prime}}^{\vartheta_{h, k}^{\prime \prime}}=\int_{-1 / k\left(k+k_{1}\right)}^{1 / k\left(k+k_{2}\right)}=\int_{-1 / k N}^{1 / k N}-\int_{-1 / k N}^{-1 / k\left(k+k_{1}\right)}-\int_{1 / k\left(k+k_{2}\right)}^{1 / k N}=\int_{-1 / k N}^{1 / k N}+E_{1}+E_{2} .
$$

The errors $E_{i}$ introduced by including these symmetrizing pieces are negligible:

$$
\begin{aligned}
\left|E_{i}\right| & =\left|\int_{1 / k\left(k+k_{i}\right)}^{1 / k N} \frac{e^{(\pi z / k)(n-1 / 24)+\pi z^{-1} / 24 k}}{z^{1 / 2}} d \phi\right| \\
& <\left(\frac{1}{k N}-\frac{1}{k\left(k+k_{i}\right)}\right) \max _{z}\left\{\frac{e^{(\pi / k) \Re(z)(n-1 / 24)+(\pi / 24 k) \Re\left(z^{-1}\right)}}{z^{1 / 2}}\right\} .
\end{aligned}
$$

Here $\Re(z)=k / n$ and

$$
\begin{aligned}
\Re\left(z^{-1}\right) & =\frac{k / n}{k^{2} / n^{2}+k^{2} \phi^{2}}<\frac{k / n}{k^{2} / n^{2}+1 /\left(k+k_{i}\right)^{2}}<k . \\
\left|E_{i}\right| & <\frac{C}{k N}\left(k^{2} / n^{2}+1 /\left(k+k_{i}\right)^{2}\right)^{-1 / 4}<\frac{C}{k n^{1 / 4}} \quad(i=1,2) .
\end{aligned}
$$

Complete the substitution $z=k / n-k i \phi$, and, making use of (5.1), write

$$
I_{h, k}=\frac{1}{k i} \int_{k / n-i / N}^{k / n+i / N} \frac{1}{z^{1 / 2}} e^{(\pi z / k)(n-1 / 24)+\pi z^{-1} / 24 k} d z+O\left(k^{-1} n^{-1 / 4}\right) .
$$

Close the path of integration by including the smaller arc of the circle $\Gamma$ through $z=k / n \pm i / N$ and tangent to the imaginary axis at the origin of the $z$-plane. We shall show this introduces a negligible error. If $z=u+i v$, the circle $\Gamma$ is given by

$$
u^{2}+v^{2}=a_{k} u, \quad 2>a_{k}=k / n+n /\left(N^{2} k\right)>1 / k .
$$

On the whole circle,

$$
\Re\left(z^{-1)}=\frac{u}{u^{2}+v^{2}}=\frac{1}{a_{k}}<k .\right.
$$

And on the smaller arc, $\Re(z) \leqq k / n$. Hence on the upper half of the smaller arc (let $s=\operatorname{arc~length}$ on $\Gamma$ ):

$$
\begin{aligned}
\left|\frac{1}{k i} \int_{\mathrm{r}}^{k / n+i / N} \frac{1}{z^{1 / 2}} e^{(\pi / k) z(n-1 / 24)+\pi z^{-1} / 24 k} d z\right| & <\frac{C_{1}}{k} \int_{z=0}^{z=k / n+i / N}|z|^{-1 / 2} d s \\
& <C k^{-1} n^{-1 / 8} .
\end{aligned}
$$

The lower half of the smaller arc of $\Gamma$ is treated in the same way. Combining 
(5.2) and (5.3) we have

$$
I_{h, k}=\frac{1}{k i} \int_{\Gamma} \frac{1}{z^{1 / 2}} e^{(\pi z / k)(n-1 / 24)+\pi z^{-1} / 24 k} d z+O\left(k^{-1}\right),
$$

the linear path of the integration used in (5.2) being replaced by the larger arc of the circle $\Gamma$. To integrate, put $t=\pi z^{-1} /(24 k)$. Then $\Re(t)=(\pi / 24 k) \Re\left(z^{-1}\right)$ $=\pi /\left(24 k a_{k}\right)=c_{k}$.

$$
I_{h, k}=\frac{2 \pi}{k}\left(\frac{\pi}{24 k}\right)^{1 / 2} \frac{1}{2 \pi i} \int_{c_{k}-i \infty}^{c_{k}+i \infty} t^{-3 / 2} e^{t+\alpha / t} d t+O\left(k^{-1}\right),
$$

where $\alpha=\pi^{2} /\left(24 k^{2}\right)(n-1 / 24)$.

Using the Hankel integral formula:

$$
\frac{1}{2 \pi i} \int_{c_{k}-i \infty}^{c_{k}+i \infty} t^{-3 / 2} e^{t+\alpha / t} d t=\sum_{0}^{\infty} \frac{\left(\alpha^{1 / 2}\right)^{2 m}}{m ! \Gamma(m+3 / 2)}=\frac{1}{(\pi \alpha)^{1 / 2}} \sinh 2 \alpha^{1 / 2} .
$$

Then

$$
\begin{aligned}
I_{h, k} & =\frac{2 \pi}{k}\left(\frac{\pi}{24 k}\right)^{1 / 2} \frac{1}{(\pi \alpha)^{1 / 2}} \frac{e^{2 \alpha^{1 / 2}}-e^{-2 \alpha^{1 / 2}}}{2}+O\left(k^{-1}\right) \\
& =\frac{e^{(\pi / k)((1 / 6)(n-1 / 24)) 1 / 2}}{(k(n-1 / 24))^{1 / 2}}+O\left(k^{-1}\right) .
\end{aligned}
$$

With this, (4.9) becomes

$$
\begin{aligned}
A(n)= & \sum_{(h, 2 k)=1,0<|h| \leqq k \leqq N,(h+k) \neq 0} e^{-\pi i(h / k) \epsilon_{\epsilon_{h, k}}} \frac{e^{(\pi / k)((1 / 6)(n-1 / 24)) 1 / 2}}{2(k(n-1 / 24))^{1 / 2}} \\
& +O\left(n^{1 / 2} \log n\right) .
\end{aligned}
$$

(On summation over $h, k$ the error terms $O\left(k^{-1}\right)$ in (5.4) give an error of $O(N)=O\left(n^{1 / 2}\right)$.)

For $k>1$, put

$$
\lambda(k)=\frac{1}{2} \sum_{(h, 2 k)=1,-k<h<k} e^{-\pi i(h / k) n} \epsilon_{h, k} .
$$

in agreement with the statement of Theorem II. For $k=1$,

$$
\lambda(1)=\frac{1}{2} e^{-\pi i n} \epsilon_{1,1}=\frac{1}{2}(-1)^{n-1} .
$$

Then from (5.5),

$$
A(n)=\sum_{0<k<n^{1 / 2}} \frac{\lambda(k) e^{(\pi / k)((1 / 6)(n-1 / 24)) 1 / 2}}{(k(n-1 / 24))^{1 / 2}}+O\left(n^{1 / 2} \log n\right) .
$$


This completes the proof of Theorem II. It should be noted that we can actually take fewer terms of the finite sum than indicated by the upper limit $\left[n^{1 / 2}\right]$ in (5.7) without increasing the order of the error term, since in fact the last term is $O\left(\lambda\left(\left[n^{1 / 2}\right]\right) / n^{3 / 4}\right)=O\left(n^{-1 / 4}\right)$. It can be shown that the number of terms taken may be reduced to $\left[n^{1 / 2} / \log n\right]$.

6. Precision of the formula. While the order of magnitude of the error term in (5.7) is small compared with that of the principal term, we were surprised to find even better agreement than expected between exact values of $A(n)$ and estimates computed from the asymptotic formula. For $n=100$ we take $\left[n^{1 / 2}\right]=10$ terms:

$$
\begin{array}{r}
-18532.888 \\
16.476 \\
-4.085 \\
.970 \\
-.402 \\
.411 \\
-.390 \\
.323 \\
-.240 \\
-.381 \\
\hline-18 \quad 520.206
\end{array}
$$

The true value is

$$
A(100)=-18520 .
$$

It is noted that after the first four terms, the error remains less than 0.5 .

For $n=200$, we take $\left[n^{1 / 2}\right]=14$ terms:

$$
\begin{aligned}
& -2660-175.054 \\
& 165.981 \\
& 5.895 \\
& -2.587 \\
& -822 \\
& -1.079 \\
& 0.000 \\
& -.227 \\
& .281 \\
& -.458 \\
& .000 \\
& .039 \\
& .184 \\
& .000 \\
& -2660007.847
\end{aligned}
$$


The exact value is

$$
A(200)=-2660008 .
$$

(Six terms are sufficient to give $A(200)$ to the nearest integer.)

TABLE I: $A(n)$

\begin{tabular}{|c|c|c|c|c|c|}
\hline 0 & 1 & 34 & -154 & 68 & -2361 \\
\hline 1 & 1 & 35 & 171 & 69 & 2525 \\
\hline 2 & -2 & 36 & -179 & 70 & -2736 \\
\hline 3 & 3 & 37 & 197 & 71 & 2937 \\
\hline 4 & -3 & 38 & -221 & 72 & -3121 \\
\hline 5 & 3 & 39 & 245 & 73 & 3333 \\
\hline 6 & -5 & 40 & -262 & 74 & -3592 \\
\hline 7 & 7 & 41 & 279 & 75 & 3856 \\
\hline 8 & -6 & 42 & -314 & 76 & -4097 \\
\hline 9 & 6 & 43 & 349 & 77 & 4367 \\
\hline 10 & -10 & 44 & -369 & 78 & -4696 \\
\hline 11 & 12 & 45 & 398 & 79 & 5034 \\
\hline 12 & -11 & 46 & -446 & 80 & -5340 \\
\hline 13 & 13 & 47 & 486 & 81 & 5683 \\
\hline 14 & -17 & 48 & -515 & 82 & -6105 \\
\hline 15 & 20 & 49 & 557 & 83 & 6521 \\
\hline 16 & -21 & 50 & -614 & 84 & -6916 \\
\hline 17 & 21 & 51 & 671 & 85 & 7365 \\
\hline 18 & -27 & 52 & -715 & 86 & -7882 \\
\hline 19 & 34 & 53 & 767 & 87 & 8409 \\
\hline 20 & -33 & 54 & -845 & 88 & -8919 \\
\hline 21 & 36 & 55 & 920 & 89 & 9473 \\
\hline 22 & -46 & 56 & -977 & 90 & -10123 \\
\hline 23 & 51 & 57 & 1046 & 91 & 10795 \\
\hline 24 & -53 & 58 & -1148 & 92 & -11429 \\
\hline 25 & 58 & 59 & 1244 & 93 & 12133 \\
\hline 26 & -68 & 60 & -1321 & 94 & -12952 \\
\hline 27 & 78 & 61 & 1421 & 95 & 13775 \\
\hline 28 & -82 & 62 & -1544 & 96 & -14580 \\
\hline 29 & 89 & 63 & 1667 & 97 & 15466 \\
\hline 30 & -104 & 64 & -1778 & 98 & -16468 \\
\hline 31 & 118 & 65 & 1898 & 99 & 17503 \\
\hline 32 & -123 & 66 & -2060 & 100 & -18520 \\
\hline 33 & 131 & 67 & 2225 & & \\
\hline
\end{tabular}

Table I gives the true values of $A(n)$ for $n \leqq 100$. These were computed by a recursion formula arising from the identity

$$
f(q)=\prod_{r=1}^{\infty}\left(1-q^{r}\right)^{-1}\left\{1+4 \sum_{m=1}^{\infty} \frac{(-1)^{m} q^{m(3 m+1) / 2}}{1+q^{m}}\right\} .
$$

From the pentagonal number theorem 


$$
\begin{aligned}
\sum_{r=-\infty}^{\infty}(-1)^{r} q^{r(3 r+1) / 2} \sum_{\nu=0}^{\infty} A(\nu) q^{\nu} & \\
= & 1+4 \sum_{m=1}^{\infty}(-1)^{m} q^{m(3 m+1) / 2} \sum_{\lambda=0}^{\infty}(-1)^{\lambda} q^{m \lambda} \\
& =\sum_{n=0}^{\infty} \gamma(n) q^{n},
\end{aligned}
$$

say, whence

$$
A(n)=\gamma(n)-\sum_{r \geqq 1}(-1)^{r}\left\{A\left(n-\frac{1}{2} r(3 r-1)\right)+A\left(n-\frac{1}{2} r(3 r+1)\right)\right\} .
$$

The coefficients $\gamma(n)$ are small and can be computed readily.

If desired, $A(n)$ may be computed directly from tabulated partition numbers $p(n)[2]$. By (6.1),

$$
\sum_{\nu=0}^{\infty} A(\nu) q^{\nu}=\sum_{r=0}^{\infty} p(r) q^{r} \sum_{m=0}^{\infty} \gamma(m) q^{m}
$$

whence

$$
A(n)=\sum_{r=0}^{n} p(r) \gamma(n-r) .
$$

This equation was used to check Table I and to compute the isolated case $A$ (200).

For the application of the asymptotic formula for $A(n)$, Table II gives values of $\lambda(k)$ for $k=1,2, \cdots, 14$, computed from (1.6) and (5.6).

7. Analogues to main theorems. With virtually no changes in the argument, analogues to Theorems I and II can be proved for the other mock theta functions of third order. This is indicated by restating the auxiliary lemmas and main theorems for the other cases $\left({ }^{3}\right)$. We make full use of the identities (1.2) and the transformation formulas (3.2), (3.3).

LEMmA 5. Let $q=\rho e^{\pi i h / k}$ and let $\rho \rightarrow 1-$. Then for fixed $h$ and $k$ with $(h, k)=1$,

$$
\begin{aligned}
\phi(q) & =O(1) \\
\psi(q) & =O(1) \\
\nu(q) & =O(1) \\
\omega(q) & =O(1)
\end{aligned}
$$

$$
\begin{array}{r}
(k \text { odd }), \\
(h \text { odd }), \\
(h+k \text { odd }), \\
(h \text { odd }) .
\end{array}
$$

(3) Once $f$ and $\omega$ are known, $\chi$ and $\rho$ are accessible from relations (1.2b, c). They are of slightly different type from the five other functions, inasmuch as their asymptotic representation in exponential form at the "worst" singular points exp $(\pi i h / k)$ does not have the same form for all $k$, as is the case for $\phi, \psi, \nu, \omega$ in Theorem III. 


$$
\begin{aligned}
& \lambda(1)=(-1)^{n-1} \frac{1}{2} \\
& \lambda(2)=\cos \left(\frac{n}{2}+\frac{3}{8}\right) \pi \\
& \lambda(3)=\cos \left(\frac{n}{3}-\frac{5}{18}\right) \pi \\
& \lambda(4)=-\cos \left(\frac{n}{4}+\frac{1}{16}\right) \pi+\cos \left(\frac{3 n}{4}+\frac{7}{16}\right) \pi \\
& \lambda(5)=\cos \left(\frac{n}{5}+\frac{2}{5}\right) \pi-\cos \left(\frac{3 n}{5}\right) \pi \\
& \lambda(6)=\cos \left(\frac{n}{6}-\frac{19}{72}\right) \pi+\cos \left(\frac{5 n}{6}+\frac{37}{72}\right) \pi \\
& \lambda(7)=-\cos \left(\frac{n}{7}+\frac{1}{14}\right) \pi+\cos \left(\frac{3 n}{7}-\frac{3}{14}\right) \pi+\cos \left(\frac{5 n}{7}-\frac{3}{14}\right) \pi \\
& \lambda(8)=\cos \left(\frac{n}{8}+\frac{13}{32}\right) \pi+\cos \left(\frac{3 n}{8}+\frac{11}{32}\right) \pi+\cos \left(\frac{5 n}{8}-\frac{11}{32}\right) \pi-\cos \left(\frac{7 n}{8}-\frac{13}{32}\right) \pi \\
& \lambda(9)=\cos \left(\frac{n}{9}-\frac{7}{.27}\right) \pi-\cos \left(\frac{5 n}{9}-\frac{2}{27}\right) \pi-\cos \left(\frac{7 n}{9}+\frac{2}{27}\right) \pi \\
& \lambda(10)=-\cos \left(\frac{n}{10}+\frac{3}{40}\right) \pi-\cos \left(\frac{3 n}{10}+\frac{1}{8}\right) \pi-\cos \left(\frac{7 n}{10}+\frac{1}{8}\right) \pi+\cos \left(\frac{9 n}{10}+\frac{27}{40}\right) \pi \\
& \lambda(11)=\cos \left(\frac{n}{11}+\frac{9}{22}\right) \pi+\cos \left(\frac{3 n}{11}-\frac{7}{22}\right) \pi+\cos \left(\frac{5 n}{11}-\frac{3}{22}\right) \pi+\cos \left(\frac{7 n}{11}+\frac{7}{22}\right) \pi \\
& +\cos \left(\frac{9 n}{11}-\frac{3}{22}\right) \pi \\
& \lambda(12)=\cos \left(\frac{n}{12}-\frac{37}{144}\right) \pi-\cos \left(\frac{5 n}{12}+\frac{19}{144}\right) \pi+\cos \left(\frac{7 n}{12}-\frac{91}{144}\right) \pi+\cos \left(\frac{11 n}{12}-\frac{35}{144}\right) \pi \\
& \lambda(13)=-\cos \left(\frac{n}{13}+\frac{1}{13}\right) \pi+\cos \left(\frac{3 n}{13}+\frac{6}{13}\right) \pi-\cos \left(\frac{5 n}{13}\right) \pi-\cos \left(\frac{7 n}{13}-\frac{2}{13}\right) \pi \\
& +\cos \left(\frac{9 n}{13}+\frac{6}{13}\right) \pi-\cos \left(\frac{11 n}{13}+\frac{2}{13}\right) \pi \\
& \lambda(14)=\cos \left(\frac{n}{14}+\frac{23}{56}\right) \pi-\cos \left(\frac{3 n}{14}+\frac{1}{56}\right) \pi+\cos \left(\frac{5 n}{14}+\frac{15}{56}\right) \pi-\cos \left(\frac{9 n}{14}-\frac{1}{56}\right) \pi \\
& +\cos \left(\frac{11 n}{14}-\frac{15}{56}\right) \pi-\cos \left(\frac{13 n}{14}-\frac{9}{56}\right) \pi
\end{aligned}
$$

Consequently, by (1.2), under the hypotheses of Lemma 5,

$$
\begin{array}{rlrl}
\phi(q)-f(-q) & =O(1) & & (h \text { odd, } k \text { even }), \\
\psi(q)+f(-q) / 2 & =O(1) & (h \text { even, } k \text { odd }), \\
\nu(q)-G_{2}(q) & =O(1) & & (h \text { odd, } k \text { odd }),
\end{array}
$$




$$
\begin{aligned}
& q^{1 / 2} \omega(q)+G_{2}\left(q^{1 / 2}\right) / 2=O(1) \quad(h / 2 \text { odd, } k \text { odd }), \\
& q^{1 / 2} \omega(q)-G_{2}\left(q^{1 / 2}\right) / 2=O(1) \quad(h / 2 \text { even, } k \text { odd }),
\end{aligned}
$$

where $G_{2}(q)$ is defined below as a product of ordinary theta functions.

Lemma 6. If $G_{2}(q)=q^{-1 / 4} \vartheta_{2}(0, q) \prod_{r=1}^{\infty}\left(1+q^{2 r}\right)$ and if $\omega_{h, k}$ is given by (2.10), (7.3) $G_{2}\left(e^{\pi i(h+i z) / k}\right)=\frac{1}{(2 z)^{1 / 2}} \delta_{h, k} e^{\pi z / 3 k+\pi z^{-1} / 24 k}\left\{1+\sum_{m=1}^{\infty} c_{h, k}(m) e^{-(\pi / k z) m}\right\}$ where $\delta_{h, k}=\omega_{h, k}^{2} / \omega_{2 h, k}^{3}$ and $\sum c_{h, k}(m) x^{m}$ is a certain convergent series $|x|<1$.

The linear transformation formulae for the functions are given by

TheOREM III. Let $(h, k)=1$, and $h h^{\prime} \equiv-1(\bmod k)$. For all complex $z$ such that $|\Im(z)| \leqq 1 /(k+1)$,

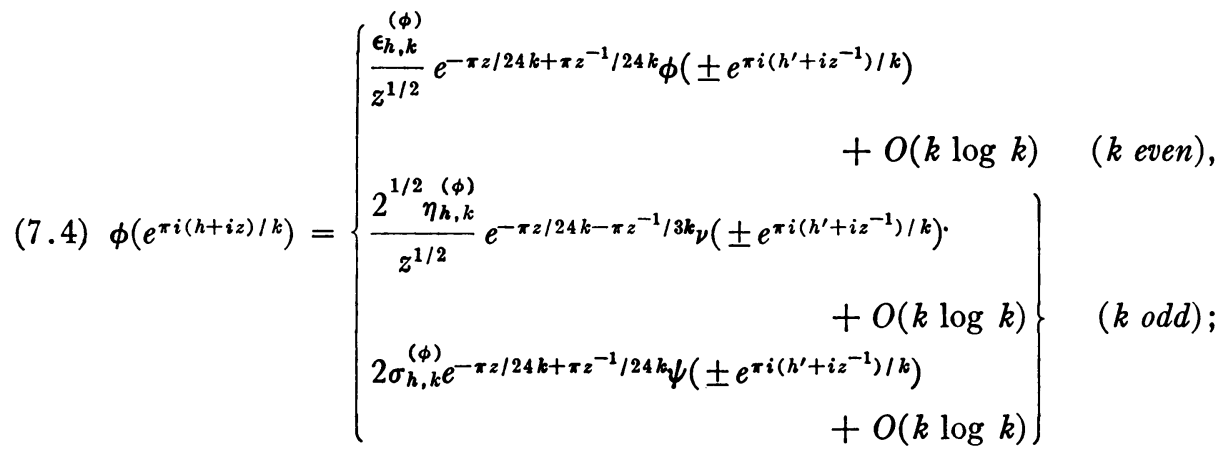

(For given $h$ and $k$, the process outlined in Lemma 4 will determine explicitly which form prevails, the value of the roots of unity $\epsilon_{h, k}^{(\phi)}, \eta_{h, \mathbf{k}}^{(\phi)}$ or $\sigma_{h, \boldsymbol{k}}^{(\phi)}$, and resolve the ambiguity of sign in the argument of the mock theta functions in the right member. In particular (7.8) gives this information in case $e^{x i h / k}$ is a boundary point for which any particular function is unbounded on radial approach.)

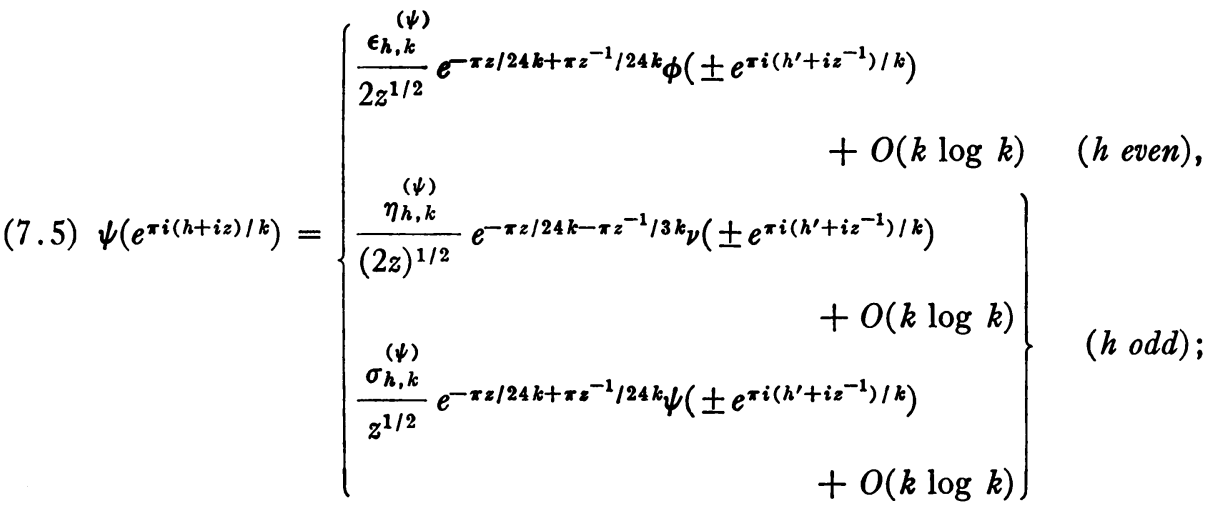




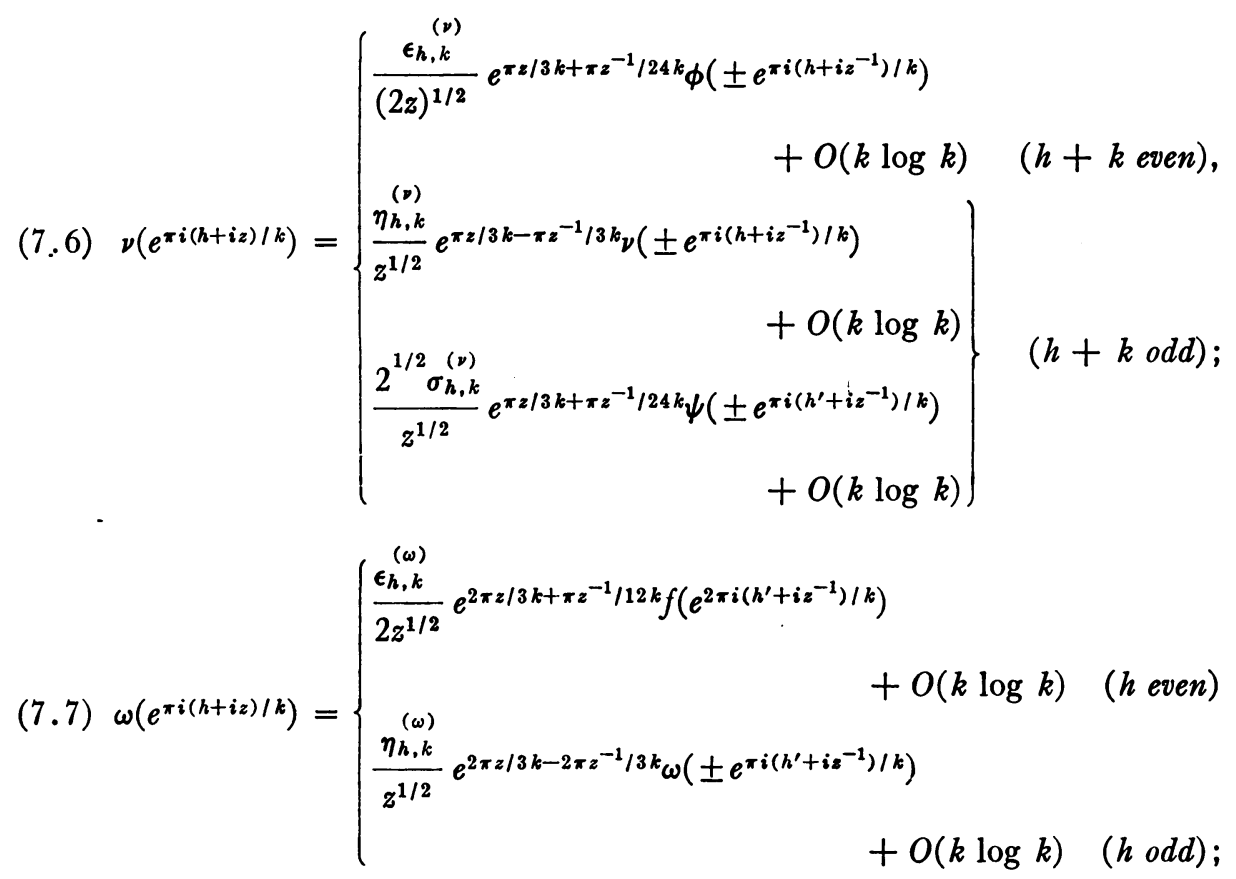

where $\left|\epsilon_{h, k}\right|=\left|\eta_{h, k}\right|=\left|\sigma_{h, k}\right|=1$ in each case, and in particular,

$$
\begin{array}{lllrl}
\epsilon_{h, k}^{(\phi)} & =\epsilon_{h-k, k} & (k \text { even }), & \epsilon_{h, k}^{(v)}=\delta_{h, k} & (h+k \text { even }), \\
\epsilon_{h, k}^{(\psi)}=-\epsilon_{h-k, k} & (h \text { even }), & \epsilon_{h, k}^{(\omega)}=(-1)^{h / 2} \delta_{h / 2, k} & (h \text { even }) .
\end{array}
$$

(See definitions (1.6) and (7.3) for $\epsilon_{h, k}$ and $\delta_{h, k}$.)

The coefficients of the power series representations of the functions are given by:

ThEOREM IV. Let $M(q)$ be one of the functions $\phi, \psi, \nu, \omega$. Then if $M(q)$ $=\sum A^{(M)}(n) q^{n}$

$$
\begin{aligned}
& A^{(\phi)}(n)=\sum_{0<k \leqq n^{1 / 2}, 2 \mid k} \lambda^{(\phi)}(k) \frac{e^{(\pi / k)((1 / 6)(n-1 / 24)) 1 / 2}}{(k(n-1 / 24))^{1 / 2}}+O\left(n^{1 / 2} \log n\right), \\
& \lambda^{(\phi)}(k)=\frac{1}{2} \sum_{-k<h<k,(h, 2 k)=1} e^{-\pi i(h / k) n} \epsilon_{h-k, k} ; \\
& A^{(\psi)}(n)=\sum_{0<k \leqq n^{1 / 2},(k, 2)=1} \lambda^{(\psi)}(k) \frac{e^{(\pi / k)((1 / 6)(n-1 / 24)) 1 / 2}}{2(k(n-1 / 24))^{1 / 2}}+O\left(n^{1 / 2} \log n\right), \\
& \lambda^{(\psi)}(k)=\frac{1}{2} \sum_{-k<h<k,(h, k)=1,2 \mid h}\left\{-e^{\left.-\pi i(h / k){ }^{\prime} \epsilon_{h-k, k}\right\} ;}\right.
\end{aligned}
$$




$$
\begin{aligned}
& A^{(v)}(n)=\sum_{\theta<k \leqq n^{1 / 2},(k, 2)=1} \lambda^{(\nu)}(k) \frac{e^{(\pi / k)((1 / 6)(n+1 / 3)) 1 / 2}}{(2 k(n+1 / 3))^{1 / 2}}+O\left(n^{1 / 2} \log n\right), \\
& \lambda^{(v)}(k)=\frac{1}{2} \sum_{-k<h<k,(h, 2 k)=1} e^{-\pi i(h / k) n} \delta_{h, k} ; \\
& A^{(\omega)}(n)=\sum_{0<k \leqq n^{1 / 2},(k, 2)=1} \lambda^{(\omega)}(k) \frac{e^{(\pi / k)((1 / 3)(n+2 / 3)) 1 / 2}}{(k(n+2 / 3))^{1 / 2}}+O\left(n^{1 / 2} \log n\right), \\
& \lambda^{(\omega)}(k)=\frac{1}{2} \sum_{-k<h<k,(h, k)=1,2 \mid h} e^{-\pi i(h / k) n}(-1)^{h / 2} \delta_{h / 2, k} .
\end{aligned}
$$

The proof of Theorem IV is virtually the same as that of Theorem II.

\section{BIBLIOGRAPHY}

1. S. Ramanujan, Collected papers, pp. 354-355.

2. S. Ramanujan and G. H. Hardy, Asymptotic formulae in combinatory analysis, Collected Papers of S. Ramanujan, pp. 276-309. 55-80.

3. G. N. Watson, The mock theta functions (I), J. London Math. Soc. vol. 11 (1936) pp. 304.

4. - The mock theta functions (II), Proc. London Math. Soc. vol. 42 (1937) pp. 274-

University of Pennsylvania,

Philadelphia, Pa. 